\title{
Analysis and Comparison of Simulation Techniques for Silver Superlenses
}

\author{
$\underline{\text { Ciaran P Moore }}^{1,2}$, Matthew D Arnold ${ }^{1,3}$, Philip J Bones ${ }^{2}$, and Richard J Blaikie ${ }^{1,2, *}$ \\ ${ }^{1}$ MacDiarmid Institute for Advanced Materials and Nanotechnology \\ ${ }^{2}$ Department of Electrical and Electronic Engineering, University of Canterbury, Christchurch, New Zealand \\ ${ }^{3}$ Current address: Physics and Advanced Materials, University of Technology Sydney, Sydney, Australia \\ *Email: r.blaikie@elec.canterbury.ac.nz \\ Telephone: +64 (3) 364-7001, Fax: +64 (3) 364-2761
}

\begin{abstract}
Transfer-matrix and finite element modelling techniques were used to simulate single- and multi-layer silver-based superlenses. The techniques were compared for their abilities to simulate subdiffraction-limited resolution and DC transmission. The finite element modelling technique confirmed conclusions drawn from T-matrix analysis, namely that multi-layer superlenses had greater transmission over a larger window of spatial frequencies than single-layer superlenses and that superlens performance was adversely affected by resonances at different frequencies. The failure of the T-matrix technique to model interactions between the mask and lens was identified as one of the main sources of inaccuracy; however, the technique remained valuable due to its superior computational efficiency compared to finite element modelling.
\end{abstract}

Keywords-superlens; near-field imaging; simulation tools.

\section{INTRODUCTION}

Super-resolving planar lenses (superlenses) are a relatively new technology, first proposed by John Pendry in 2000 [1]. They work by enhancing and coupling surface plasmon polaritons (SPPs) across thin layers of silver, interleaved with layers of dielectric. This results in the transmission of subwavelength information over greater distances (and at higher intensities) than would normally be available from conventional propagation (Fig. 1). The first optical wavelength superlenses were demonstrated near simultaneously by two separate groups in 2005 [2,3]. They used $50 \mathrm{~nm} \mathrm{[2]} \mathrm{and} 35 \mathrm{~nm} \mathrm{[3]} \mathrm{thick} \mathrm{silver} \mathrm{films} \mathrm{(Fig.} \mathrm{2)}$ illuminated with transverse magnetic (TM) polarised $365 \mathrm{~nm}$ wavelength UV light to resolve $72.5 \mathrm{~nm}$ and $60 \mathrm{~nm}$ half-pitch features, respectively. These remarkable achievements resulted in further research on possible superlens designs and geometries, with papers published that detailed multi-layer [4], patterned [5] and perforated [6] geometries.

Due to the small scales $(\leqslant \mu \mathrm{m})$ and complex fabrication processes involved, robust simulation techniques have become an essential tool for the design of superlenses and other superresolving systems, such as left-handed metamaterials (LHMs). Several methods, such as finite difference time domain (FDTD) analysis [e.g. 2,7], multiple

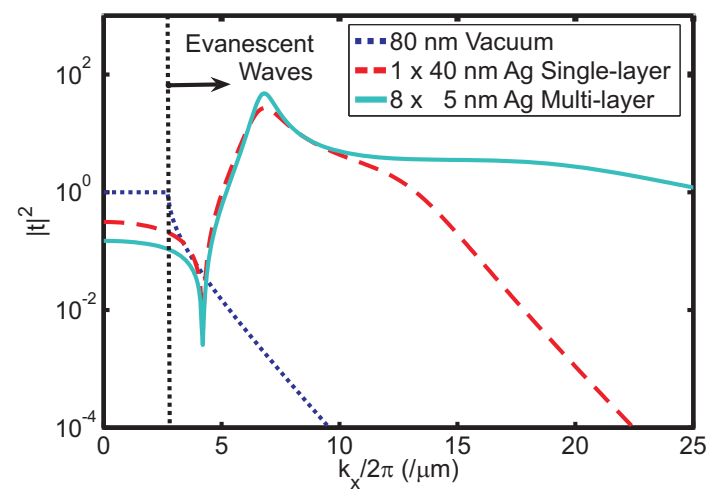

Figure 1. Transfer functions for single- and multi-layer superlenses and an $80 \mathrm{~nm}$ vacuum gap.

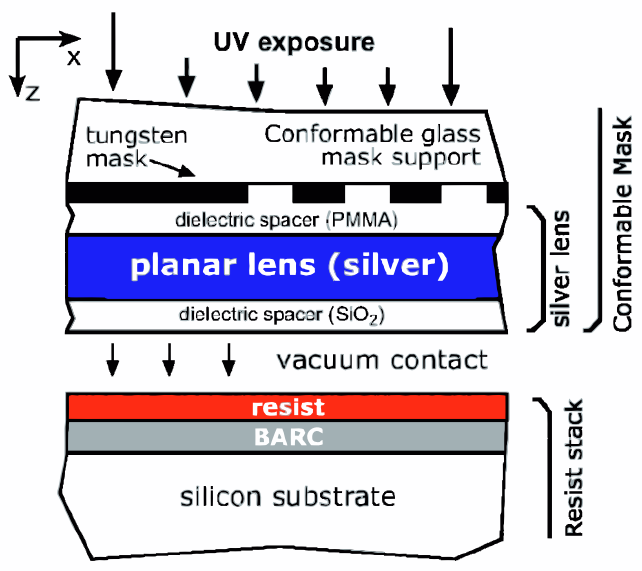

Figure 2. Typical superlens construction [2].

multipole programming [8] and finite element modelling (FEM) $[9,10,11]$ were used successfully in the past; however, they were all relatively computationally intense and could be slow to produce solutions. Hence, a computationally efficient transfer matrix (T-matrix) method was developed [12] to give an estimate of lens performance using only a few, relatively simple calculations. Preliminary results [10] showed that the T-matrix approximations were in general agreement with fullfield simulations, allowing them to be used in subsequent 
work [13]. The purpose of this paper is to examine the relationship between the approximations given by the $\mathrm{T}$ matrix technique and the more accurate results obtained from FEM, explaining any differences that appear. The particulars of both modelling systems are given, before examples of individual results are offered for comparison.

\section{Simulation TECHNiQUeS}

The T-matrix technique modeled lenses as a series of material interfaces, each with entrance and exit relative permittivities (Fig. 3). In a similar way to the calculation of scattering parameters for RF applications [e.g. 14], transmission and reflection coefficients were calculated for the interface from $2 \times 2$ transfer matrices,

$$
\left[\begin{array}{c}
E_{x}^{C} \\
E_{x}^{D}
\end{array}\right]=\frac{k_{z}^{\prime \prime}}{2 \varepsilon^{\prime \prime}}\left[\begin{array}{cc}
\frac{\varepsilon^{\prime \prime}}{k_{z}^{\prime \prime}}+\frac{\varepsilon^{\prime}}{k_{z}^{\prime}} & \frac{\varepsilon^{\prime \prime}}{k_{z}^{\prime \prime}}-\frac{\varepsilon^{\prime}}{k_{z}^{\prime}} \\
\frac{\varepsilon^{\prime \prime}}{k_{z}^{\prime \prime}}-\frac{\varepsilon^{\prime}}{k_{z}^{\prime}} & \frac{\varepsilon^{\prime \prime}}{k_{z}^{\prime \prime}}+\frac{\varepsilon^{\prime}}{k_{z}^{\prime}}
\end{array}\right]\left[\begin{array}{c}
E_{x}^{A} \\
E_{x}^{B}
\end{array}\right]=T(0)\left[\begin{array}{c}
E_{x}^{A} \\
E_{x}^{B}
\end{array}\right],
$$

and were then referred to the exit $(z=d)$ plane,

$$
T(d)=\left[\begin{array}{cc}
\exp \left(-i k_{z}^{\prime \prime} d\right) & 0 \\
0 & \exp \left(i k_{z}^{\prime \prime} d\right)
\end{array}\right] T(0)\left[\begin{array}{cc}
\exp \left(i k_{z}^{\prime} d\right) & 0 \\
0 & \exp \left(-i k_{z}^{\prime} d\right)
\end{array}\right] \text {. }
$$

Multiplying them together gave a transfer matrix for the entire lens stack,

$$
\left[\begin{array}{l}
t \\
0
\end{array}\right]=\left[\begin{array}{ll}
T_{11} & T_{12} \\
T_{21} & T_{22}
\end{array}\right]\left[\begin{array}{l}
1 \\
r
\end{array}\right]
$$

which was used to find the spatial-frequency transfer function of a lens (Fig. 1). Lens behaviour was simulated by convolving the transfer function with different input waveforms (Fig. 4). A more comprehensive explanation of the T-matrix technique can be found in Ref. 10.

Although T-matrix calculations were computed to a high degree of accuracy, they were inherently limited because interactions between the lens and the mask above it were not considered [12]. In contrast, the FEM method - implemented using the COMSOL Multiphysics package [15] - was a fullfield simulation technique that took account of mask-lens interactions, giving solutions to Maxwell's equations over geometries similar to the one shown in Fig. 2. Relative permittivity, $\varepsilon_{r}$, and permeability, $\mu_{r}$, values were specified for the mask material and for each layer in the lens stack, allowing generation of solutions (Fig. 5) for several different physical properties. In a similar way to the T-matrix simulations, TM polarised light was used at a wavelength of $365 \mathrm{~nm}$.

\section{RESULTS}

Previous analysis [13] performed using the T-matrix technique showed that multi-layer superlenses [4] had better transmission at higher wave numbers $\left(k_{x} / 2 \pi\right)$ than equivalent single-layer superlenses (Fig. 1). It was also shown that lens performance could be application specific, due to varying DC attenuation across lenses and lens-specific resonances that

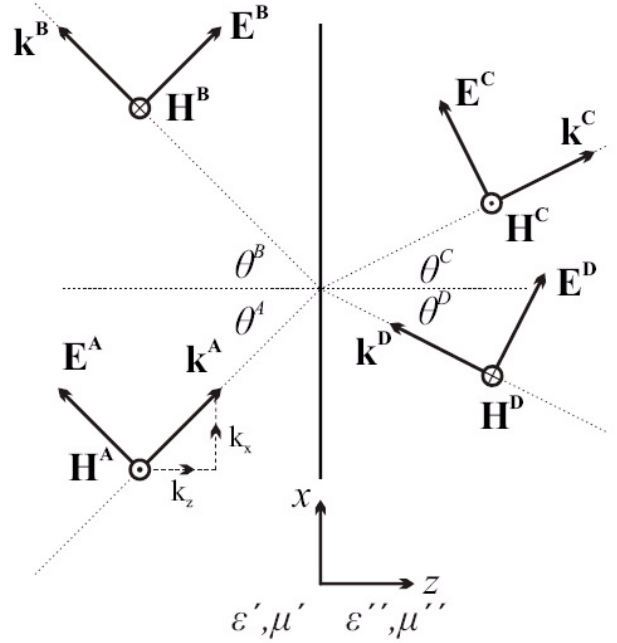

Figure 3. Domain setup for T-matrix simulations [12]
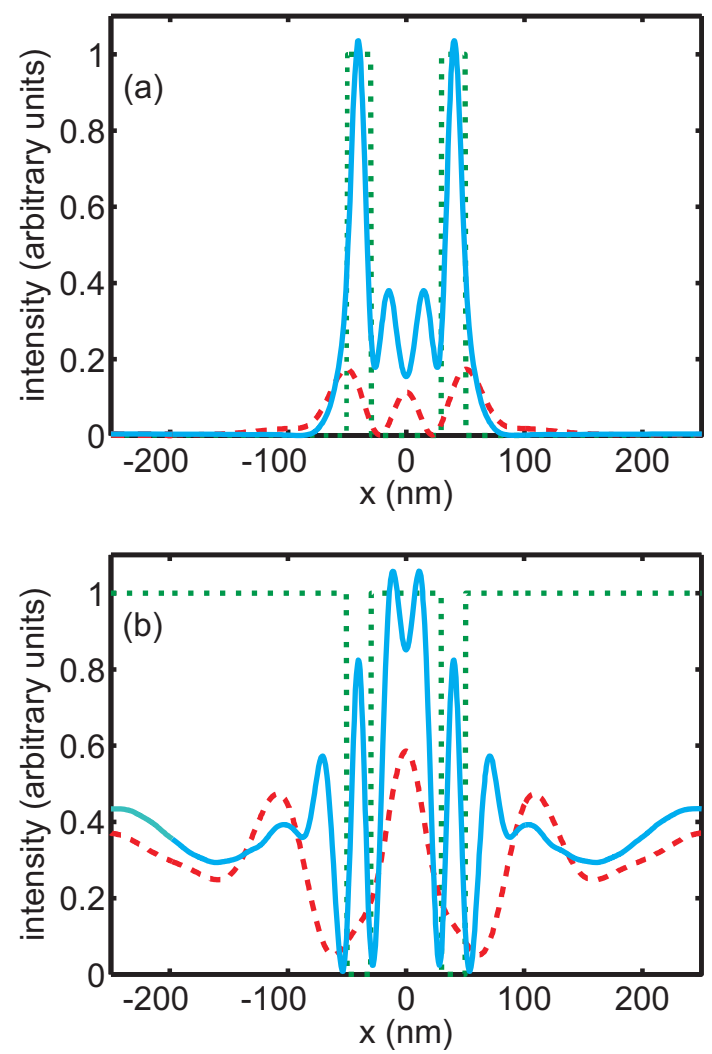

Figure 4. T-matrix simulation of $20 \mathrm{~nm}$ dual feature input patterns (dotted) imaged through single- (dashed) and multi-layer (solid) superlenses.

occurred at certain spatial frequencies. For instance, a multilayer lens outperformed its single layer equivalent when imaging $20 \mathrm{~nm}$ bright features (Fig. 4(a)) but not when imaging equivalent dark features (Fig. 4(b)).

Lastly, image inversion and frequency doubling in the output waveforms were identified using performance metrics derived 


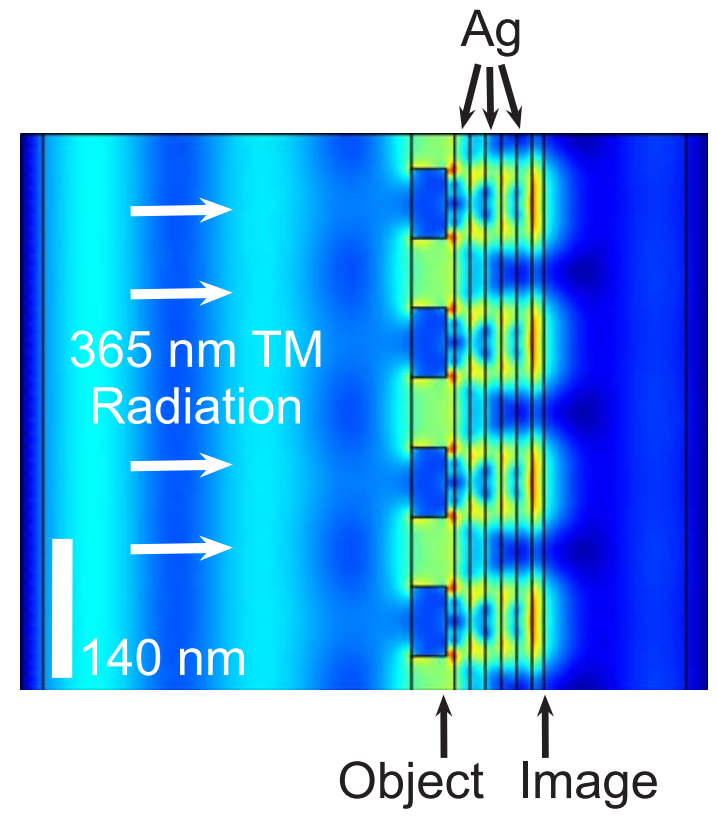

Figure 5. FEM simulation of $140 \mathrm{~nm}$ period grating imaged through a triplelayer superlens, made up of three $13.3 \mathrm{~nm}$ thick silver laminations.

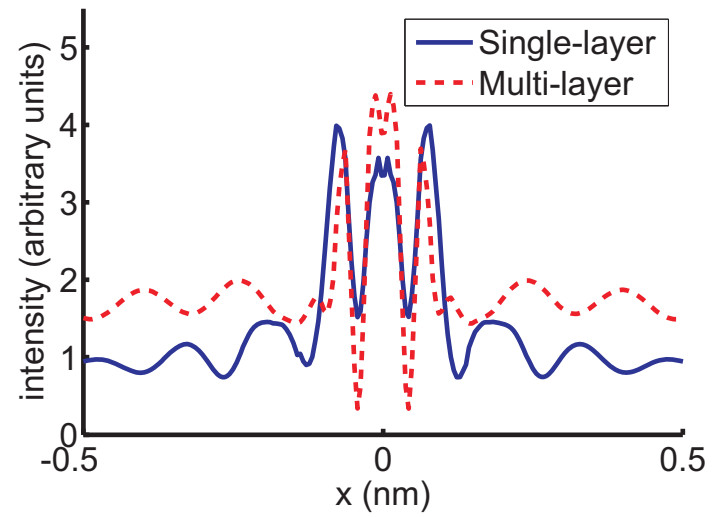

Figure 6. FEM simulation of a $20 \mathrm{~nm}$ dual dark feature mask imaged through single- and multi-layered superlenses.

from the simulation data. The general validity of these findings is confirmed here via FEM simulations and details of differences are presented.

Firstly, steeper gradients in FEM-simulated results confirmed that multi-layer lenses had better transmission at higher wave numbers compared to single-layer lenses (Fig. 6) and that lens performance was affected by frequency-dependent resonances (Fig. 7). In this case, image intensity was much greater for a $400 \mathrm{~nm}$ period object (Fig. 7(b)) compared with similar $300 \mathrm{~nm}$ and $500 \mathrm{~nm}$ period objects (Fig. 7(a) and (c)), for both FEM and T-Matrix simulations. These resonances were identified at both the object and the image planes, confirming that mask-lens interactions could significantly alter lens performance. The same image metrics [13] applied to the T-matrix-generated data were calculated for the FEM results. Once again, these identified instances of frequency doubling and image inversion, as shown in the pseudo-contrast analysis in Fig. 8.
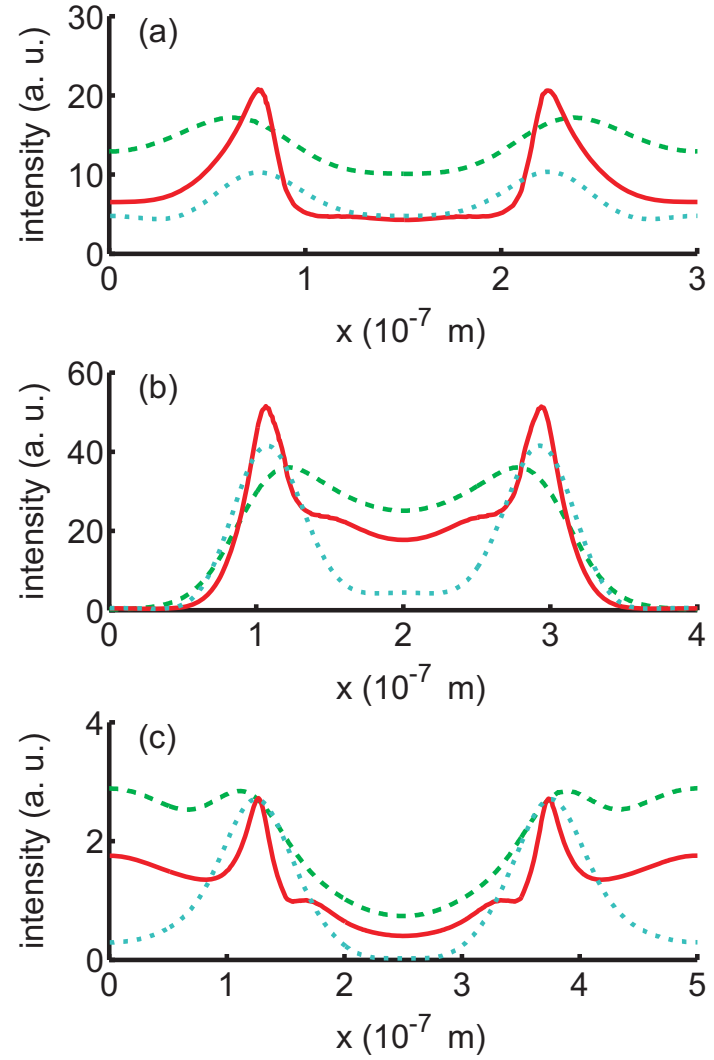

Figure 7. FEM (dashed) and T-matrix (dotted) simulations of (a) $300 \mathrm{~nm}$, (b) $400 \mathrm{~nm}$ and (c) $500 \mathrm{~nm}$ period cosine-like masks (solid) imaged through an ideal, single-layer superlens.

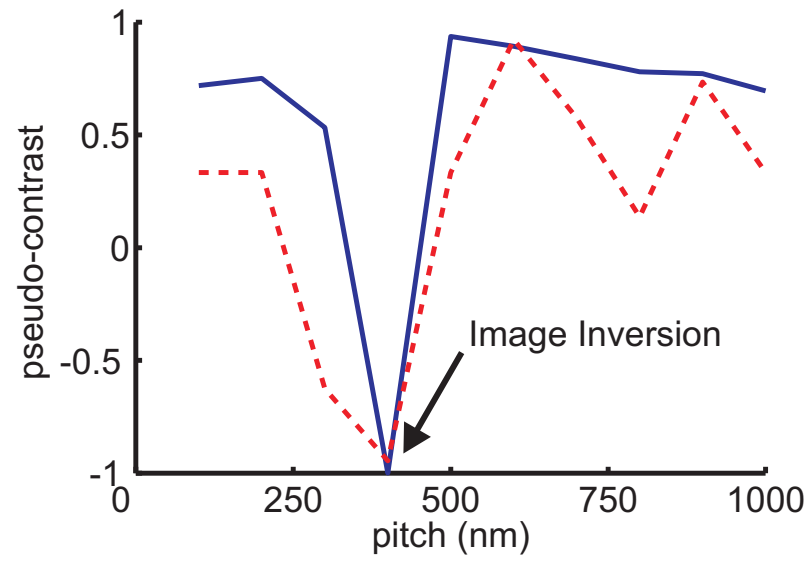

Figure 8. FEM (solid) and T-matrix (dashed) simulated pseudo-contrast profiles for gratings imaged through an ideal, single-layer superlens. Note: pseudo-contrast is defined in Ref. 13. Higher pseudo-contrast is better and negative pseudo-contrast indicates image inversion.

In an effort to improve the T-matrix technique, mask input data was captured from FEM simulations and convolved with T-matrix derived superlens transfer functions. These replaced the ideal, step-like input profiles that had previously been used for T-matrix simulations [13] and led to closer agreement between T-matrix and FEM output profiles (Fig. 9). Despite this, the conclusion that multi-layer superlenses had lower DC transmission than single-layer equivalents, which was 

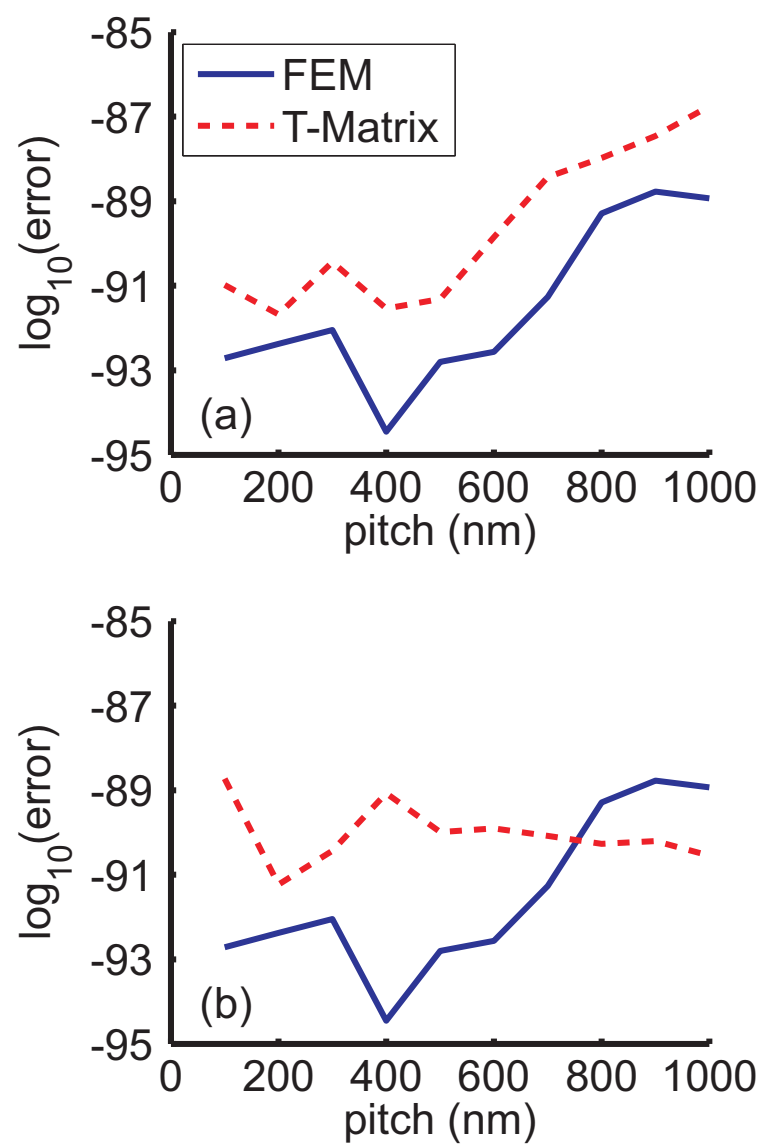

Figure 9. FEM (solid) and T-matrix (dashed) simulated error profiles for gratings imaged through a realisable, single-layer superlens [2]. FEM curves are identical in (a) and (b), however, T-matrix curves are based on (a) FEMgenerated and (b) ideal mask profiles. Note: error is defined in Ref. [13]. Lower error is better.

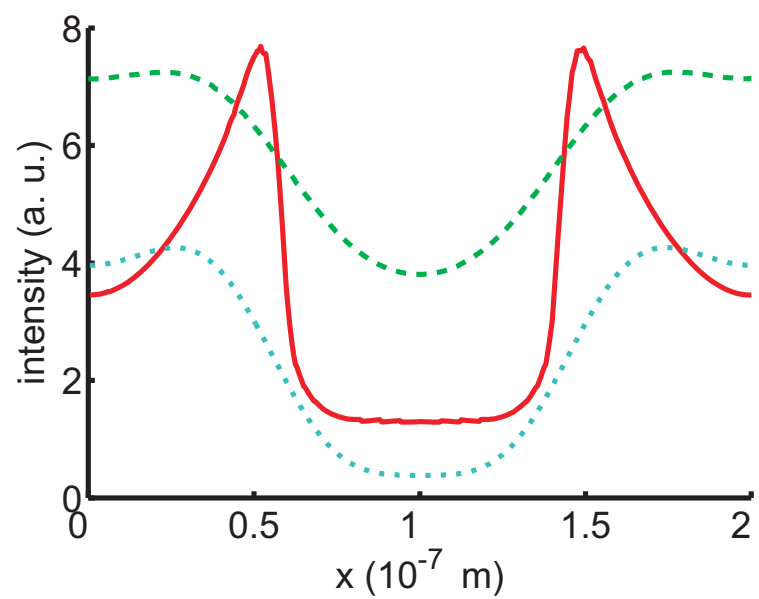

Figure 10. FEM (dashed) and T-matrix (dotted) simulations of a $200 \mathrm{~nm}$ period mask (solid) imaged through an ideal, single-layer superlens.

suggested by analysis of T-matrix data, could not be confirmed by FEM. Indeed, comparison of FEM and T-matrix data indicated that, although both techniques often produced results that were qualitatively similar, they seldom agreed on their estimation of the DC term (Fig. 10). This suggested that mask-lens interactions, which were accounted for by FEM but not by the T-matrix technique, played a significant role in the determination of lens performance above the diffraction limit.

\section{CONCLUSION}

Several silver-based superlens designs were simulated using an approximate transfer matrix technique and a full-field iterative FEM solver. Agreement was found between equivalent simulations; however, several shortcomings were exposed in the T-matrix technique, such as DC coefficient mismatch due to neglect of interactions between the mask and the superlens. Using FEM-derived input profiles with the Tmatrix technique improved some aspects of the T-matrix simulations but did not resolve the differences in DC levels between the two methods, indicating that further work is required to address the problem of mask-lens interaction in superlens applications.

\section{REFERENCES}

[1] J. B. Pendry, "Negative refraction makes a perfect lens," Phys. Rev. Lett., 85, pp. 3966-3969, October 2000.

[2] D. O. S. Melville and R. J. Blaikie, "Super-resolution imaging through a planar silver lens," Opt. Exp. 13, pp. 2127-2134, March 2005.

[3] N. Fang, H. Lee, C. Sun and X. Zhang, "Sub-diffraction-limited optical imaging with a silver superlens," Science 308, pp. 534-537, April 2005.

[4] D. O. S. Melville and R. J. Blaikie, "Experimental comparison of resolution and pattern fidelity in single- and double-layer planar lens lithography," J. Opt. Soc. Am. B 23, pp. 461-467, March 2006.

[5] Z. Liu et al., "Far-field optical superlens," Nano Lett. 7, pp. 403-408, January 2007.

[6] P.-Y. Chen and S.-C. Wu, "A periodic porous-nanostructured lefthanded material slab at optical frequencies," Microwave Opt. Technol. Lett. 50, pp. 216-220, January 2008.

[7] S. A. Cummer, "Simulated causal subwavelength focusing by a negative refractive index slab,” Appl. Phys. Lett. 82, pp. 1503-1505, March 2003.

[8] R. J. Blaikie and S. J. McNab, "Simulation study of 'perfect lenses' for near-field optical nanolithography," Microelec. Eng. 61-62, pp. 97-103, July 2002.

[9] D. Schurig et al., "Metamaterial Electromagnetic Cloak at Microwave Frequencies," Science 314, pp. 977-980, November 2006.

[10] D. O. S. Melville, "Planar lensing lithography: enhancing the optical near field," $\mathrm{PhD}$ Thesis, University of Canterbury, New Zealand, February 2006.

[11] A. V. Kildishev et al., "Comment on "Negative refractive index in artificial metamaterials [Opt. Lett. 31, pp. 2483-2485, July 2006],” Opt. Lett. 32, pp. 1510-1511, June 2007.

[12] D. O. S. Melville and R. J. Blaikie, "Analysis and optimization of multilayer silver superlenses for near-field optical lithography," Phsica B 394, pp. 197-202, May 2007.

[13] C. P. Moore et al., "Image Fidelity for Single- and Multi-Layer Silver Superlenses," J. Opt. Soc. Am. A, 25, pp. 911-918, February 2008.

[14] G. J. Rogers, D. E. Sawyer and R. L. Jesch, "Semiconductor Measurement Technology: Measurement of Transistor Scattering Parameters," U. S. Department of Commerce / National Bureau of Standards Special Publication \# 400-5, Washington, D.C., January 1975.

[15] COMSOL is a registered trademark of COMSOL AB, (C) 1997-2008. 